\title{
High-Resolution Broadband Millimeter-Wave Astrophysical Spectrometer with Triple Product Acousto-Optical Processor
}

\author{
Miguel Chavez Dagostino, Alexandre S. Shcherbakov, Adan Omar Arellanes, Vahram Chavushyan \\ National Institute for Astrophysics, Optics \& Electronics (INAOE), Puebla, Mexico \\ Email: mchavez@inaoep.mx, alex@inaoep.mx, arellaneso@inaoep.mx, vahram@inaoep.mx
}

Received October 10, 2013; revised November 5, 2013; accepted November 12, 2013

Copyright (C) 2013 Miguel Chavez Dagostino et al. This is an open access article distributed under the Creative Commons Attribution License, which permits unrestricted use, distribution, and reproduction in any medium, provided the original work is properly cited.

\begin{abstract}
An advanced conceptual design of a high-bit-rate triple product acousto-optical processor is presented that can be applied in a number of astrophysical problems. We briefly describe the Large Millimeter Telescope as one of the potential observational infrastructures where the acousto-optical spectrometer can be successfully used. A summary on the study of molecular gas in relatively old (age $>10 \mathrm{Myr}$ ) disks around main sequence stars is provided. We have identified this as one of the science cases in which the proposed processor can have a big impact. Then we put forward triple product acousto-optical processor is able to realize algorithm of the space-and-time integrating, which is desirable for a wideband spectrum analysis of radio-wave signals with an improved resolution providing the resolution power of about $10^{5}$. $10^{6}$. It includes 1D-acousto-optic cells as the input devices for a 2D-optical data processing. The importance of this algorithm is based on exploiting the chirp Z-transform technique providing a 2D-Fourier transform of the input signals. The system produces the folded spectrum, accumulating advantages of both space and time integrating. Its frequency bandwidth is practically equal to the bandwidth of transducers inherent in acousto-optical cells. Then, similar processor is able to provide really high frequency resolution, which is practically equal to the reciprocal of the CCD-matrix photo-detector integration time. Here, the current state of developing the triple product acousto-optical processor in frames of the astrophysical instrumentation is shortly discussed.
\end{abstract}

Keywords: Astrophysical Instrumentation; Radio-Astronomy; Millimeter-Wave Spectrometer; Space-and-Time Integrating; Acousto-Optical Devices; Optical Processing System

\section{Introduction}

Molecular astrophysics, sometimes also called astrochemistry, has a long history. Molecules in space have been studied panchromatically since the discovery of diffuse interstellar bands nearly a century ago in the optical spectral range [1,2]. At UV wavelengths, the spectroscopic capabilities of the Copernicus satellite launched in the early 70 s conduced to the detection of molecular rotational transitions of nitrogen, hydrogen and some other more complex molecules including carbon monoxide $[3,4]$. Almost contemporarily, the first attempts to identify molecules at (sub)-millimeter wavelengths, which is the interval we are particularly interested in, resulted in the detection of $\mathrm{CO}$ and $\mathrm{CN}[5,6]$.

Molecules are present in virtually every astrophysical scenario: Interstellar, circumstellar and pregalactic gas, circumnuclear gas in active galactic nuclei, the atmospheres of stars and planets of the solar system, and very recently in exoplanetary atmospheres [7]. They provide valuable information on gas properties such as excitation, kinematics and chemistry and are amongst the main coolant agents that help on the collapse of primordial material to form galaxies and interstellar clouds to form new stellar generations. While for the analysis of extragalactic objects a rather coarse resolution is enough due to the Doppler broadening (velocity dispersion) of the spectral lines ( $230 \mathrm{MHz}$ or $300 \mathrm{~km} / \mathrm{s}$ at $230 \mathrm{GHz}$ ), galactic studies require a significantly enhanced resolution, of the order of a few $\mathrm{KHz}(0.05 \mathrm{~km} / \mathrm{s})$.

The construction of (sub)-millimeter spectrometers has been usually based on filter banks and auto-correlators, nevertheless, for a long time it has been recognized the numerous advantages offered by acousto-optic technique, 
among others, stability, low weight, compactness, and relatively low cost. In fact, these advantages have prompted the construction of acousto-optical spectrometers, not only for a number of earth based and air-borne (sub)-millimeter telescopes [8-11], but also for space missions such as the heterodyne instrument on board of Herschel [12].

So far, nearly 170 circumstellar and interstellar molecular species have been identified, accounting for billions of transitions (vibrational, rotational and electronic) that provide an enormous richness at every spectral band [13]. In connection with these problems of modern molecular astrophysics, the next step can be made in application of the acousto-optical processors to astrophysical radio-wave observations at $1.3 \mathrm{~mm}(230 \mathrm{GHz})$. In this paper, we present an advanced conceptual design of a high-bit-rate triple product acousto-optical processor that would complement the near future expected instrumental capabilities of the Large Millimeter Telescope. The Large Millimeter Telescope Alfonso Serrano (GTM-AS/ LMT-AS).

The LMT-AS is a bi-national collaboration between Mexico and the USA, led by the National Institute for Astrophysics, Optics, and Electronics (INAOE) and the University of Massachusetts at Amherst, to construct, commission and operate a $50 \mathrm{~m}$-diameter millimeterwave radio telescope [14]. Construction of the telescope structure is complete at the $4600 \mathrm{~m}$ LMT site on the summit of Volcán Sierra Negra (Puebla, Mexico). Firstlight and Early Science observations with the LMT were successfully conducted, respectively, in June-July 2011 and May-July of 2013. Within these observing runs the telescope operated with the inner 32 meter diameter of the antenna and the premier instruments AZTEC, a continuum camera sensitive at $1.1 \mathrm{~mm}$ and the Redshift Search Receiver, a spectrometer capable of obtaining spectral images in a wide band $(80-110 \mathrm{GHz})$ at a resolution of about $40 \mathrm{MHz}$. The reader is referred to the LMT webpage (www.lmtgtm.org) for more details on the telescope and its current instrumentation. A third instrument, SEQUOIA, was successfully used at the $14 \mathrm{~m}$ antenna of the five college observatory in Massachusetts and is expected to be part of the available instrumentation for the LMT-AS starting in the winter of 2014.

SEQUOIA is a 32-element heterodyne focal plane array receiver for the $85-115 \mathrm{GHz}$ frequency band. The system makes use of MMIC (Monolithic Microwave Integrated Circuit) preamplifiers and covers $15 \mathrm{GHz}$ of instantaneous bandwidth. The instrument was used at the FCRAO 14 m antenna between 1998 and 2006 to carry out wide field imaging of molecular line emission in the Milky Way and nearby galaxies [15]. Despite its age, SEQUOIA is still the largest, most sensitive $3 \mathrm{~mm}$ focal plane array available today. The instrument will be up- graded in 2014, by replacing the noisiest pixels, and expanding its bandwidth. It is our intent to field the instrument on the 32-m LMT in the winter of 2014, where SEQUOIA will benefit from higher-quality atmospheric conditions, 4 times larger collecting-area (4 times improved surface brightness sensitivity) and 2 times better angular resolution compared to its previous successful observations on the FCRAO 14-m.

\section{Studies of Gassy Disks around Normal Main Sequence Stars}

There are numerous astrophysical niches in which a high resolution spectrograph operating within relatively wide frequency bandwidth is a fundamental tool. They are related mainly to the formation of structures as well as stars in their advanced evolutionary stages. The following brief explanation describes one of the most interesting potential applications for similar acousto-optical radio-wave spectrographs.

The global picture in the formation of planetary systems starts with a primordial or protoplanetary disk. Young stellar objects are usually surrounded by material composed of gas and dust. In the very early stages this gas-left over from the original gas cloud that gave rise to star formation-vastly dominates the circumstellar material with only a few percent of the total mass being in the form of dust. As the disk evolves, the gas is removed from the system through several processes, among which, photoevaporation and viscous accretion, leaving the systems dominated by small dust particles that produce the infrared excesses observed in main sequence stars $[16,17]$. Whilst the gaseous material is expected to be depleted when the star-disk systems is only a few million year old, far less that the stellar ages, there are a few stellar examples in which gas is still present in observable amounts, even for relatively old systems.

The atomic gas component has been optically detected in only a few debris disks, with the most prominent examples being those of the stars Beta-Pictoris and HD32297 [18] thanks to the edge-on orientation of the circumstellar material. Molecular gas, on the other side, has been more elusive and has been ascertained in only two systems, 49 Ceti and HD21997, however, in amounts that vastly supersede that of atomic gas, as large as 14 Earth masses for the star 49 Ceti $[19,20]$.

The current debate is whether in these systems, like 49 Ceti, the gas is indeed a residual from the primordial gas that survived the early depletion process or corresponds to a second generation material, in a similar way the dust is thought to be replenished by the collision of planetesimals [21]. The answer to this controversy will certainly come out from sensitive millimeter wave observations at an increased resolution aimed at detecting molecular gas (primarily of $\mathrm{CO}$ ) that would be eventually 
complemented with optical observation of atomic gas. To date, there are only a few attempts to find protoplanetary systems similar to 49 Ceti, and the confirmation of the existence of gas and its ubiquity in such systems will await the availability of high-resolution instruments on large aperture facilities such as the Large Millimeter Telescope.

For the special case described above and the more general scenario of star formation, it is necessary to count with a large bandwidth and high resolution, feasible with acousto-optical techniques. As previously indicated, a large bandwidth would allow the inclusion of many molecular species, hence, very valuable tools for constraining the physical properties of the gas. In particular, we propose the application of acousto-optic techniques for the development of a highly productive radio-wave spectrometer with a bandwidth of analysis lying in the gigahertz frequency range together with a spectral resolution belonging to the kilohertz frequency range to work at 1.3 $\mathrm{mm}(230 \mathrm{GHz})$. The above-mentioned spectral resolution and frequency bandwidth can be evidently realized by the triple product acousto-optical processor exploiting a space-and time integrating [22]. Such an approach provides the resolution power of about $10^{5}-10^{6}$, which makes it possible to investigate not only the intense emission of the carbon monoxide molecule, ${ }^{12} \mathrm{CO}(2-1)$, but also other prominent species such as sulphur dioxide $\left(\mathrm{SO}_{2}\right)$, cyanoacetylene $\left(\mathrm{C}_{3} \mathrm{HN}\right)$, and others, many of which will be studied for the first time. The expected performances of the radio-wave acousto-optical spectrometer under consideration will be potentially comparable to that of the Atacama Large Millimeter Array (ALMA), and the highest ever achieved in a single dish telescope that will certainly take millimeter wave spectral observations to discovery grounds.

\section{Spatially One-Dimensional Acousto-Optical Input Devices}

Generally, the systems for optical information processing have an advantage to be three-dimensional in their nature, so that the directions of propagating the corresponding light flows can be usually considered as optical axes. During the propagation of light through each of similar systems, the modulations of light flow by the input data and the other optical transformations can be performed to obtain the desirable issuing light distribution at the output plane of that system. Usually, various modulators, lenses, and other components for optical data processing are located in the planes being orthogonal to that optical axis, so that a two-dimension arrangement makes it possible to carry out a large amount of parallel operations and, in so doing, to achieve potentially ultra-high productivity of processing. Together with this, during the last years a lot of two-dimensional architectures for opti- cal data processing has been progressed, wherein various one-dimensional input devices have been exploited, while these systems as a whole have been designed in a three-dimension space. Realizing two-dimensional optical processors with one-dimensional input devices is not so easy, as a rule; nevertheless, similar systems exhibit a high level of flexibility. As a result, rather large amount of high-quality one-dimensional input devices have been created and inserted into optical systems for two-dimensional data processing in a view of creating high-bit-rate and adaptable optical processors. One of the most important arguments for applying one-dimensional input devices in two-dimensional processing is based on relatively high level of their development. The most widespread of them are, for example, linear arrays of semiconductor light sources like laser diodes or light emission diodes (LEDs); then, one can call a row of electro-optic, acousto-optic, and magneto-optic cells; various CCD linear arrays, etc. It should be noted that modern semiconductor light sources allow effective and ultra-high frequency (up to $10^{10} \mathrm{~Hz}$ ) external amplitude modulation, while one-dimensional Bragg acousto-optical cells (AOCs) provide effective conversion of electrical signal into optical ones in a wide (up to a few gigahertz) frequency bandwidth with time-bandwidth product (i.e. a product of the frequency bandwidth and the aperture transit time) exceeding $10^{3}$. Rather small sizes of similar input devices and modern technique of designing the needed optical lenses give real opportunity for constructing ultra-highproductive and compact optical systems for parallel data processing. Moreover, both relatively low levels of the needed electric power and not high prices inherent in these components lead potentially to realizing compact optical processors of low power consumption as well as of low cost.

In the case of exploiting spatially one-dimensional $\mathrm{AOC}$ as an input device, the data can be introduced in a sequential regime. If the signal applied to similar AOC is modulated by the time function $f(t)$, the light scattered by this cell will be modulated by the function

$f(t-x / V)$, where $x$ is the coordinate of acoustic wave propagation and $V$ is the acoustic wave velocity. Consequently, at each instant of time $t$, the scattered light is modulated proportionally to the input electric signal with respect to the spatial coordinate $x$; and together with this, light is modulated by the input electric signal at each individual value of the coordinate $x$ with respect to the time $t$. Such a doubled modulation can be important within designing dynamic optical processors, because in the last case the AOC can be exploited as the input devices and as the delay lines. In reality, the modulation provided by AOC is much more complicated process. The scattered light has Doppler frequency shift relative to the initial central frequency, and finite size of 
the AOC's aperture always restricts the spatial length of an area wherein the modulation of light beam can be fulfilled. Then, the proportionality between the depth of light modulation and the applied electric signal is true only when diffraction efficiency of the AOC does not exceed a few percent. Additionally, the modulating properties of AOC operating in Bragg regime are essentially depending on the angle of light incidence on a cell. All these factors should be taken into account during the design of practical systems.

The AOC can be efficiently used as a point modulator as well, because it can provide pure amplitude modulation for the light beam entirely. Within such an application of the AOC, one can imagine that the AOC's aperture is going to zero, so that space-time signal

$f(t-x / V)$ is degenerated into the time function $f(t)$. If, for example, the AOC is lighted by a conic optical beam focused near the piezoelectric transducer, the scattered optical beam represents almost the same cone modulated with respect to time by the function $f(t)$. The bandwidth of modulation is inversely proportional to the acoustic transit time through an individual focal spot. For example, with the focal spot size of about $4 \mu \mathrm{m}$ and typical acoustic wave velocity $4 \cdot 10^{5} \mathrm{~cm} / \mathrm{s}$, the acoustic transit time through that focal spot has the order of $1 \mathrm{~ns}$. It should be noted that with growing the KleinCook parameter [23], which is accompanied by increasing the diffraction efficiency of AOC, the output light cone assumes an elliptic shape due to appearing wave mismatch. Nevertheless, this estimation is rather close to allowable time of direct electronic amplitude modulation for the laser diodes or LEDs. However, sometimes these light sources exhibit the radiation with too low time coherence under action of high-bit-rate modulation. This is why just the AOCs can be efficiently exploited as point modulators providing a wideband time modulation of the light beam as a whole.

Nevertheless, efficiency of exploiting the third dimension in a three-coordinate space has the decisive significance, because only this property gives potential advantages to optical systems in comparison with the other ones. Consequently, an important question appears: is a two-dimensional data processing system capable of providing rather high productivity under exploitation of just one-dimensional input devices? The adequate answer looks like this: if a one-dimensional input device is involved, some part of the functional possibilities of parallel processing becomes to be lost, because instead of about $10^{6}$ resolvable spots of data, provided usually by a spatially two-dimensional light modulator, now only $10^{3}$ spots can be realized. However, the losses in parallelism can be successfully compensated by very wide frequency bandwidth of already existing one-dimensional input devices. Let us consider a few estimations. The aperture transit time $T$ of the AOC can be undoubtedly about $30 \mu \mathrm{s}$ (see below) or even more up to $0.1 \mathrm{~ms}$. The frequency bandwidth $\Delta f$ of similar AOC is usually about $50 \mathrm{MHz}$ or more. Consequently, the time-bandwidth product can be definitely estimated as

$N=T \cdot \Delta f \geq 10^{3}$, which represents the number of potentially achievable resolvable spots. This product is almost independent on concrete magnitudes of both $T$ and $\Delta f$, because it is mainly conditioned by fundamental acousto-optical relations. The direct comparisons of different processors are not always possible, but some estimation can be done. For example, if the AOC with the product $N=T \cdot \Delta f$ is lighted by the modulated beam from a single-channel source and the frequency bandwidth of this external modulation is taken equal to $\Delta f$ as well, one yields $\Delta f \cdot N$ of multiplications per second, because during each time interval $1 / \Delta f$ one can realize $\mathrm{N}$ multiplications. In the case when a pair of similar AOC is located sequentially, the triple-product processor will perform $\Delta f \cdot N^{2}$ multiplications per second. As a result, using the above-mentioned estimations for the aperture transit time and the frequency bandwidth for the AOC, one can expect the final bit-rate about $10^{14-15} \mathrm{mul}$ tiplications per second. Thus, one can conclude that involving one-dimensional input devices with really wide frequency bandwidth in two-dimensional optical gives a chance to create potentially the prototypes of rather highbit-rate optical processors.

\section{Schemes and Algorithms for Spectrum Analyzers}

Let us consider a typical acousto-optical spectrum analyzer depicted in Figure 1(a). The acousto-optical spectrum analyzer includes the laser source of monochromatic light, which is directed to the AOC through the lens $L 1$ or another collimating optics in such a way that the needed angular requirements are satisfied. In its turn, the AOC is excited by a wide-frequency signal correlated with those angular requirements in the plane $P 1$. The integrating lens $L 2$ converts the angular spectrum into the linear one and focuses plane waves in an amount of spots lying in its focal plane. The photo-detector is represented by a multi-pixel CCD-linear array, which shapes a discrete video-signal. This scheme allows two extreme options of designing. On the one hand side, the frequency bandwidth is about $50 \mathrm{MHz}$, the processing time is about $30 \mu \mathrm{s}$, and the number of parallel channels 1500 , while on the other hand, the frequency bandwidth is about $1.0 \mathrm{GHz}$, the processing time is about $1.0 \mu \mathrm{s}$, and the number of parallel channels 1000 . Usually, performance data inherent in the majority of similar systems lee between these extreme cases with more or less the same time-bandwidth product 1000 - 1500. The number of pixels exceeds usually the time-bandwidth product by 


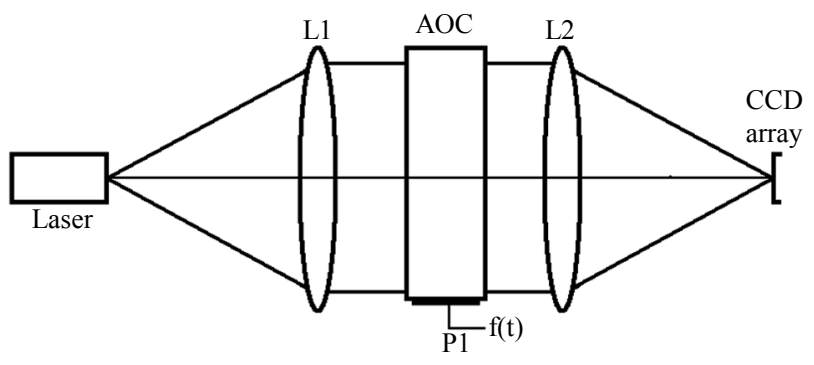

(a)

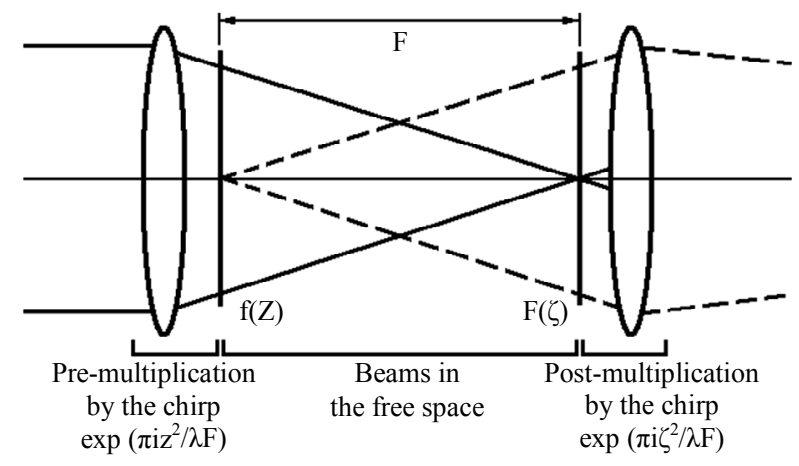

(b)

Figure 1. Optical layouts of the acousto-optic spectrum analyzers with the space integration (a) and for the chirp Z-transform (b).

2 - 3 times to provide the needed discreteness of a spectrum under analysis. Because of each doublet or maybe triplet of pixels is equivalent to a single frequency channel, the CCD-linear arrays have usually from 1024 to 4096 pixels, and the expected number of frequency channels can be really large, the advantages of acoustooptical spectrum analyzers can be easily estimated. Natural parallelism inherent in the optical system illustrates potential superiority of the acousto-optics in comparison with electronics in this area. During the operation of acousto-optical spectrum analyzer lighting the AOC is almost perpendicular to its input optical facet, and together with this the piezoelectric transducer of that AOC is exited by an external electronic signal $f(t)$, so that the light wave signal at the output facet can be finally represented as $f(z, t)$. The lens $L 2$ provides the Fourier transform $F(w, t)$ in the plane $P 2$, where

$$
F(w, t)=\int_{-\infty}^{\infty} f(x, t) \cdot \exp (2 \pi i w z) \mathrm{d} z
$$

where the space frequency $w$ is connected with the physical coordinate $\zeta$ in the plane $P 2$ and the focal distance $F$ of the integrating lens $L 2$ as

$w=\zeta /(\lambda F)$. In fact, the integration limits are constrained by the aperture of the AOC. Because of integration in Equation (1) is performed with respect of the space coordinate at the input plane of a cell, one can say that this analyzer belongs to a group of architectures with the space integrating.

The ability to resolve signals in the frequency domain is oriented on fixing the gap of a pre-scripted depth between two frequencies in the Fourier domain. Moreover, the reasons for this capability are connected with the Rayleigh criterion for resolution within determining the angular distance between stars using a telescope. The Rayleigh criterion is based on the uniformity of lighting the telescope aperture, so that light intensity in the image plane is described by the squared sinc-function. In so doing, the gap of a 1-dB-depth can be obtained with a peak magnitude for one frequency component coincides with the first zero for the neighboring frequency component. The gap of such a depth is well seen visually as well as on a photo-film. Applying the Rayleigh criterion to the acousto-optical spectrum analyzer, one can find that the spatial distance $\delta \zeta$ between two neighboring frequency components in the focal plane of the integrating lens $L 2$ can be estimated as

$\delta \zeta=\lambda F / D=\delta f \cdot \lambda F V$, where $F$ is the focal distance of the lens $L 2, D$ is the aperture of the AOC, $V$ is the acoustic velocity, and $\delta f$ is the frequency resolution. These equalities give us

$\delta f=V / D=1 /($ transit time $)$. Moreover, one can apply various techniques for the incident light apodization to detect definitely weak signals. However, the suppressing of side lobes can be achieved at the expense of the corresponding demerit in the frequency resolution.

The other algorithm for spectrum analysis can be based on the time integration and the chirp Z-transform is a method, which is often exploited for performing the Fourier transform in systems where only the time variable is available. The chirp $Z$-transform is based on the fact that the factor $2 \pi \zeta \mathrm{z} /(\lambda F)$ in the kernel of Fourier transform is equal to

$\pi\left[\zeta^{2}+z^{2}-(\zeta-z)^{2} /(\lambda F)\right]$. The equivalent way for explaining the Fourier-transform determined by Equation (1) gives

$$
\begin{aligned}
& F(w, t)=\exp \left[\pi i \zeta^{2} /(\lambda F)\right] \\
& \times \int_{-\infty}^{\infty}\left\{f(z, t) \exp \left[\pi i z^{2} /(\lambda F)\right]\right\} \exp \left[-\pi i(\zeta-z)^{2} /(\lambda F)\right] \mathrm{d} z
\end{aligned}
$$

This formula demonstrates that at first the optical signal $f(z, t)$ at the input facet is multiplied by the factor $\exp \left[\pi i z^{2} /(\lambda F)\right]$. Then, the Fresnel transform of the obtained product is fulfilled, and finally the result of integration is multiplied by the factor $\exp \left[\pi i \zeta^{2} /(\lambda F)\right]$ to compensate a square-law phase factor. The Fresnel transform in optics means that a light beam with some space distribution is propagating at a distance $F$ in free 
space. In principle, it is a physical phenomenon accompanied by a diffraction when the angle of light diffraction is linearly dependent on both time and space frequencies at the input. Figure 1(b) represents a configuration for an optical system capable of performing the chirp Z-transform. Thus one can see its analogy with the Fouriertransform system depicted in Figure 1(a); their only difference consist in the positions of lenses. The lens $L 1$ lights an external electronic signal $f(t)$ by coherent light beam, providing its pre-multiplication by the factor $\exp \left[\pi i z^{2} /(\lambda F)\right]$. The resulting beam propagation in free space does the operation of integral convolution, while the lens $L 2$ gives us the needed post-multiplication by the factor $\exp \left[\pi i \zeta^{2} /(\lambda F)\right]$. Application of the chirp Z-transform to various system architectures provides their significant flexibility relative to both the frequency resolution and the frequency bandwidth for analysis as well as makes it possible to design spectrum analyzers with the time integration, which have considerably improved frequency resolution.

\section{A Triple-Product Acousto-Optical Processor}

The technique of signal processing based on the spaceand-time-integrating had been proposed for the spectrum analysis of one-dimensional signals [24,25]. In principle, the analysis of one-dimensional signals can be provided by one-dimensional acousto-optical analyzer with only the space- or only the time-integrating. However, these analyzers have the performances supplementing with one another. The space-integrating gives a wide frequency bandwidth together with relatively low frequency resolution, while the time-integrating provides a high resolution for spectrum components accompanied by comparably narrow frequency bandwidth. A two-dimensional processor combining the advantages from both spaceand time-integrating makes it possible to obtain the analysis with wide bandwidth as well as high resolution simultaneously. A scheme of the space-and-time integrating spectrum analyzer exploiting a pair of the acousto-optical cells (AOCs) is shown in Figure 2. The triple product acousto-optical processor applies the algorithm of the space-and-time integrating, which provides rather wideband studies with an up rated spectral resolution. This processor includes a pair of mutually orthogonally oriented wide-aperture 1D-acousto-optic cells as the input devices for a 2D-optical spectrum analysis. Significance of this approach is conditioned by the usage of the above-noted chirp Z-transform technique realizing a 2D-Fourier transform of the input signal $s(t)$ [26, 27].

The laser diode or LED, for example, can be used as a point light source whose radiation is modulated in time by the initial electronic signal $\Phi_{0}(t)$. The vertically



Figure 2. Schematic arrangement for a triple-product acousto-optical processor.

oriented acousto-optic cell AOC-1 does a modulation by the first additional electronic signal $\Phi_{1}(t)$. This cell is lighted by the optical beam from a point source through the spherical lens $L 1$ and the cylindrical lens $L 2$. The light beam, scattered by the AOC-1, is modulated by the product $\Phi_{0}(t) \cdot \Phi_{1}\left(t-x_{1} / V_{1}\right)$. In a view of lighting the AOC-2, which is placed horizontally, the output light beam after the AOC-1 is broadened horizontally and focused vertically by the spherical lens $L 3$. Let the second additional electronic signal, which is applied to the AOC-2, be $\Phi_{2}(t)$. As a result, the light beam, scattered by the AOC-1, is modulated by the triple product $\Phi_{0}(t) \Phi_{1}\left(t-x_{1} / V_{1}\right) \Phi_{2}\left(t-x_{2} / V_{2}\right)$. The obtained result includes in fact two time delays $t_{1}=x_{1} / V_{1}$ and $t_{2}=x_{2} / V_{2}$, where $x_{1,2}$ and $V_{1,2}$ are physical spatial coordinates along the corresponding acousto-optical cells and the acoustic wave velocities, respectively. These time delays $t_{1}$ and $t_{2}$ must satisfy the inequality $0<\left(t_{1}, t_{2}\right)<T$, where the aperture transit time $T$ of the modern acousto-optical cells can be equal to about $10-50 \mu \mathrm{s}$. The cylindrical lens $L 4$ and the spherical lens $L 5$ shape the image of the AOC-2 at the output plane in horizontal direction, while the spherical lenses $L 3$ and L5 give the image of the AOC-1 at the output plane in vertical direction. A two-dimensional matrix of photo-detectors is placed in the output plane, so that charges $g\left(t_{1}, t_{2}\right)$ collected by each individual pixel under acting the light during the time $T_{i}$ at a point $(x, t)$ are proportional to

$$
g\left(t_{1}, t_{2}\right)=\int_{T_{i}} \Phi_{0}(t) \Phi_{1}\left(t-x_{1} / V_{1}\right) \Phi_{2}\left(t-x_{2} / V_{2}\right) \mathrm{d} t,
$$

where $T_{i}$ is the time of integration, which is limited by the detector and could be about $1 \mathrm{~ms}$ or more; the time delays $t_{1}$ and $t_{2}$ represent a pair of the coordinates in the output focal plane, i.e. in a plane of the CCD matrix photo-detector. Such a system represents a triple-product processor. However, the system does not simply collect two one-dimensional conversions; the final result appears within a joint two-dimensional processing of all the input signals. This architecture always calculates Equation (3), but it exhibits really high flexibility, because all the three input signals are given initially as electronic signals. In 
this way the processor can be easily re-oriented from fulfilling one algorithm to another in the frames of completely the same optical resources simply by varying the input electronic signals $\Phi_{j}(t)$ with $(j=0,1,2)$.

\section{Brief Description of Potential Advantages Peculiar to the Space-and-Time Integrating Processor}

Let us apply algorithm of the above-mentioned chirp$Z$-transform technique to processing the signal $s(t)$. That technique involves two frequency-chirp signals whose parameters $k_{1} x /\left(2 \pi V_{1}\right)$ and $k_{2} y /\left(2 \pi V_{2}\right)$ represent the frequency variables along the output axes $x$ and $y \quad\left(V_{1,2}\right.$ are the acoustic velocities as before). In so doing, one can take, for example: $V=V_{1}=V_{2}$, $\Phi_{0}(t)=s(t) \cdot \exp \left(i k_{1} t^{2}+i k_{2} t^{2}\right), \Phi_{1}(t)=\exp \left(-i k_{1} t^{2}\right)$, and $\Phi_{2}(t)=\exp \left(-i k_{2} t^{2}\right)$. As a result the output function will have the form:

$$
\begin{aligned}
& g(x, y)=\exp \left[-i k_{1}(x / V)^{2}-i k_{2}(y / V)^{2}\right] \\
& \times \int_{T_{i}} s(t) \exp \left[-2 i k_{1}(x / V) t-2 i k_{2}(y / V) t\right] \mathrm{d} t
\end{aligned}
$$

Without the square-law phase term, the function $g(x, y)$ can be considered as the Fourier-transform of the signal $s(t)$, which has been calculated using the algorithm of chirp-Z-transform with respect to both the space coordinates, so that spectrum of the signal $s(t)$ will be displayed along the coordinates $x$ and $y$. Maximal frequencies for the spectrum inherent in the signal $s(t)$, and presented along the axes $x$ and $y$, are equal to $k_{1} D /(2 \pi V)$ and $k_{2} D /(2 \pi V)$, where $D$ is the apertures of both the AOCs. If the factor $k_{1}$ is chosen in such a way that $k_{1} D /(2 \pi V)=\Delta f$ is the bandwidth of the signal $s(t)$, the complete frequency spectrum of this signal will be registered and represented along the axis $x$ with relatively low frequency resolution corresponding to the conventional space integration spectrum analysis. This estimation is conditioned by the fact that resolution is determined by the ratio of the frequency bandwidth $\Delta f$ to the number $M$ of pixels peculiar to the taken $M \times M \quad C C D$-matrix along the axis $x$, i.e. the frequency resolution is characterized by the value $\Delta f / M$. Additionally, if the factor $k_{2}$ is selected as $k_{1} / M$, one has arrived at the case when only a $1 / M$-part of a spectrum will be depicted along the axis $y$ for each individual position along the axis $x$. As a result, the frequency resolution along the axis $y$ becomes to be equal to the value $\Delta f / M^{2}$. These estimations are true under condition that the time of integration $T_{i}$ exceeds $M^{2} / \Delta f$. A combination of the transformation along the axis $x$ with relatively low resolution in a wide frequency bandwidth with the transformation along the axis $y$ with a high frequency resolution, but in a narrow frequency bandwidth represents a two-dimensional folded spectrum. Such a spectrum gives a two-dimensional raster for recording $M^{2}$ counts of one-dimensional Fourier-images of the signal $s(t)$. Shaping a onedimensional spectrum along a pair of space coordinates within this architecture makes it possible to display up to $M^{2}$ spectrum components, because the matrix of photodetectors has $M^{2}$ pixels. Thus, this technique provides potentially a high frequency resolution together with rather wide frequency bandwidth of the spectrum analysis. Finally, this processor produces the resultant folded spectrum, which is subject to electronic post-processing. Generally, this technique accumulates the principal advantages of both space and time integrating acousto-optical processors. In particular, with the typical value of $M=10^{3}$, similar system exhibits rather wide frequency bandwidth $\Delta f$, which is practically equal to bandwidth of the input piezoelectric transducers of acousto-optical cells. Broadly speaking, the magnitude of $\Delta f$ can be estimated from $30 \mathrm{MHz}$ to more than $1 \mathrm{GHz}$ in modern acousto-optics. Together with this, similar system is able to provide a high frequency resolution $\delta f$, which is practically equal to the reciprocal of the CCD-matrix photo-detector integration time $1 / T_{i}$. Within optimal choosing of both the integration time $T_{i}$ and the abovenoted factors $k_{1,2}$ inherent in a pair of the frequencychirp signals, one can vary the needed frequency resolution $\delta f$ from about $10 \mathrm{~Hz}$ to $10 \mathrm{KHz}$.

\section{Schematic Arrangement and Current Design}

One of the allowable practical arrangements of the above-characterized optical scheme for the triple-product acousto-optical processor is presented as a simplified prototype in Figures $\mathbf{3}$ and $\mathbf{4}$ (some optical components are not shown in this figures). The scheme is based on an advanced 3-inch components, i.e. for a spectrum analyzer with the space-and-time integrating, depicted in Figure 2. The designed arrangement is localized on a $4 \times 1$-feet optical breadboard and based on specifically selected and optimized set of three-inch spherical and cylindrical lenses. The semiconductor laser, allowing an external amplitude modulation with the frequency up to $3 \mathrm{GHz}$ can be potentially chosen as a point source of light because its light output is arranged through a connector with a span of the single mode optical fiber.

The selected optical and mechanical components make it possible to use of highly effective and specially designed wide-aperture AOCs. For this purpose, in particular, let us take a few spatially one-channel crystalline AOCs. For example, in the case of relatively low frequencies of analysis one can consider a tellurium dioxide $\left(\mathrm{TeO}_{2}\right)$ crystalline cell from Molecular Technology $\mathrm{GmbH}$, see Figure 5. It has the access time $T \approx 85 \mu \mathrm{s}$, 


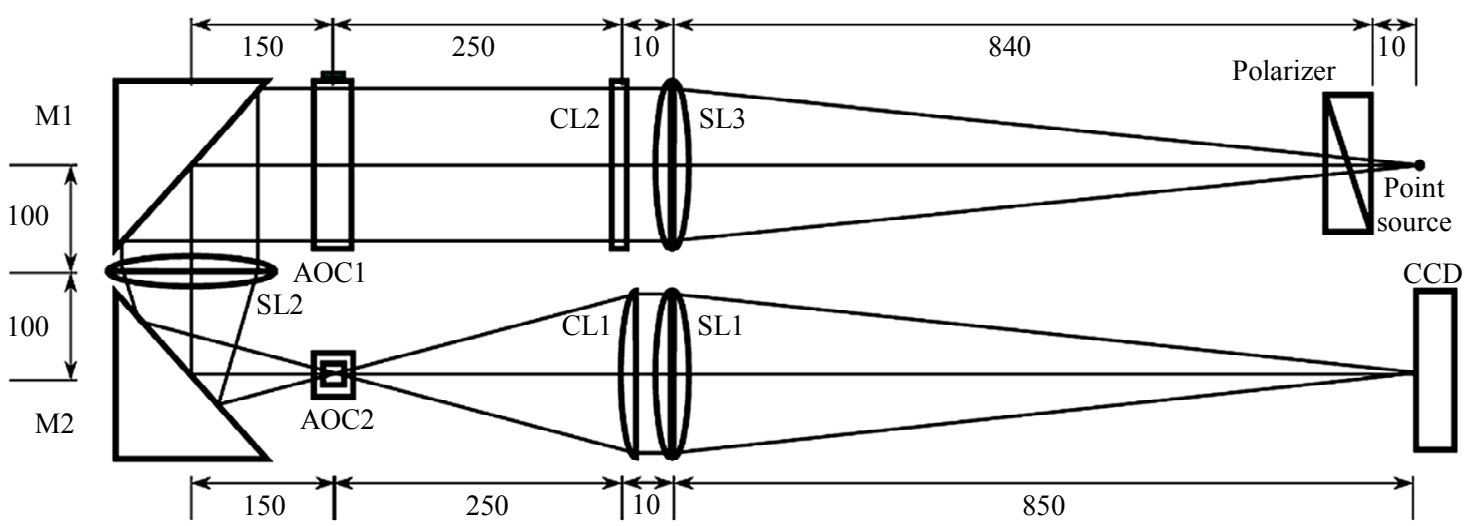

Figure 3. Layout of the simplified optical scheme for the acousto-optical triple-product processor.

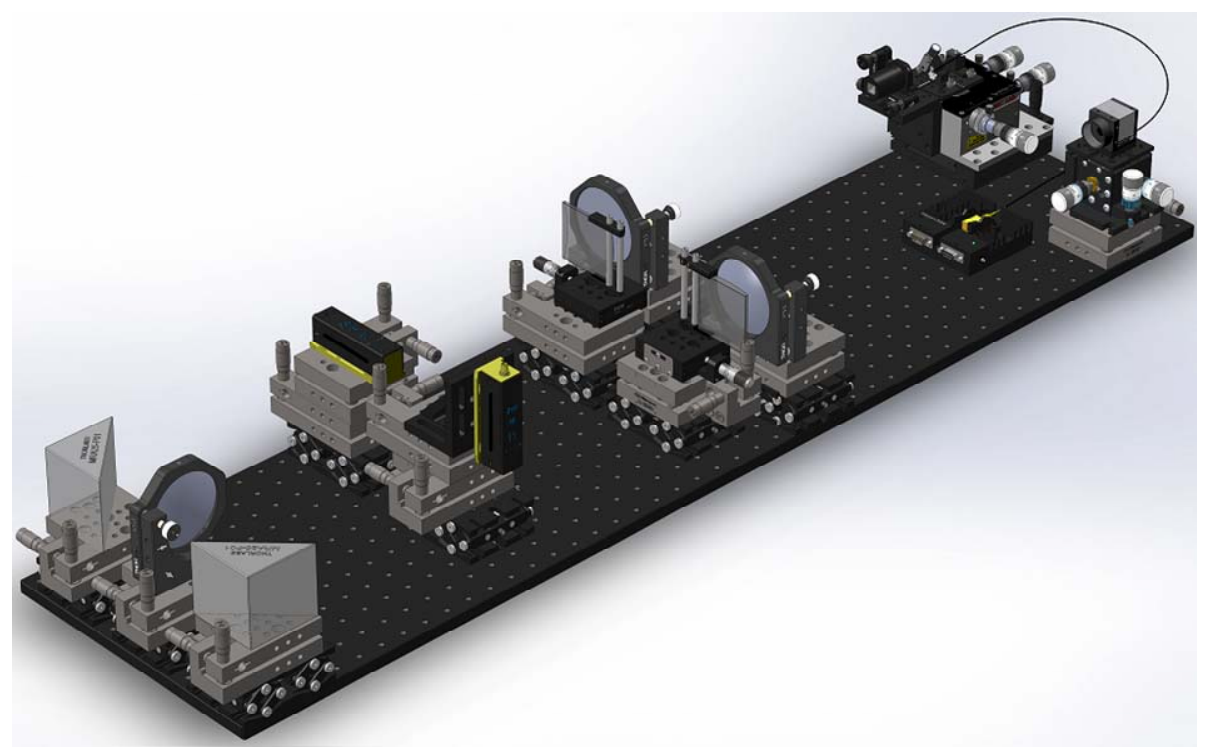

Figure 4. Potential simplified design of a prototype for the triple-product acousto-optical processor.

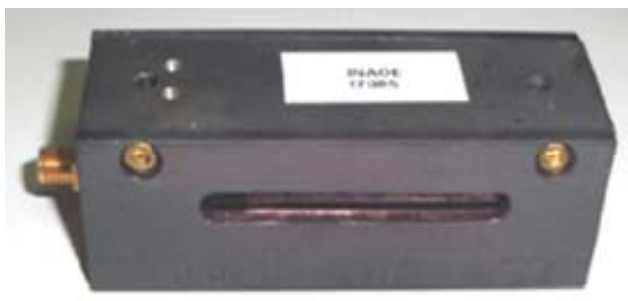

Figure 5. A $\mathrm{TeO}_{2}$-crystal based AOC. (Molecular Technology GmbH).

optical aperture $3 \times 55 \mathrm{~mm}$, diffraction efficiency $>65 \%$, and frequency bandwidth $50 \mathrm{MHz}$ at the carrier frequency $75 \mathrm{MHz}$, so that its time-bandwidth product [28] is about 4250 . Together with this, one can mention high frequency AOCs mode of lithium niobate $\left(\mathrm{LiNbO}_{3}\right)$ and rutile $\left(\mathrm{TiO}_{2}\right)$ crystals $[29,30]$. These AOCs operate in the gigahertz range up to $5 \mathrm{GHz}$ providing the frequency bandwidth of about $1-3 \mathrm{GHz}$ per one spatial channel. Their optical apertures and time-bandwidth products are mainly limited by total acoustical losses accessible per aperture for the chosen acoustical mode and the technology selected for producing the needed piezo-electric transducers.

Then, the CCD matrix camera EO-5023M (Edmund Optics) can be potentially considered as the time-integrating multi-pixel (more than $4 \times 10^{6}$ pixels of $3.45 \times$ $3.45 \mu \mathrm{m}$ ) photo-detector due to its 12-bit pixel depth and up to $60 \mathrm{~s}$ exposure time. This camera with the sensing area $8.45 \times 7.07 \mathrm{~mm}$ is presented in Figure 6 .

\section{Conclusions}

The current state of designing a high-bit-rate triple product processor has been presented. The main goal is to create an advanced acousto-optical radio-wave spectrometer for application to the molecular astrophysics. The above-presented consideration of a few key aspects related to creating an optical scheme of the processor reflects an indispensable step for the practical design of 


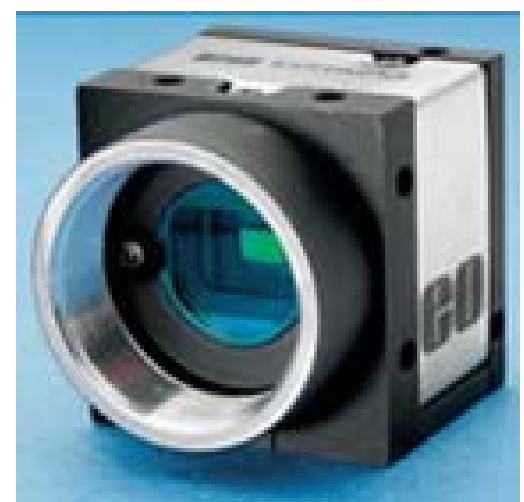

Figure 6. The CCD matrix camera EO-5023M (Edmund Optics).

similar radio-wave spectrometer. The acousto-optical processor under construction will be able to carry out the algorithm of the space-and-time integrating.

This capability is desirable for the simultaneous analysis of a wideband spectrum of radio-wave signals and the improved resolution power, expected to be about $10^{5}-10^{6}$ for operations at $1.3 \mathrm{~mm}(230 \mathrm{GHz})$. A reasonable part of the performed analysis has been devoted to practical design of the schematic arrangement currently based on three-inch optics and wide-aperture spatially one-channel AOCs as the input devices for a 2Doptical data processing. Various 1D-acousto-optical cells made of the tellurium dioxide $\left(\mathrm{TeO}_{2}\right)$, lithium niobate $\left(\mathrm{LiNbO}_{3}\right)$, and rutile $\left(\mathrm{TiO}_{2}\right)$ crystals have been briefly mentioned together with the CCD-matrix specifically oriented to an increased exposure time, i.e. to the enlarged integration time. Undoubtedly, developing such a highly productive radio-wave spectral instrument promises both appreciable progresses in the technique of precise observations associated with pioneering results in modern molecular astrophysics.

\section{Acknowledgements}

This work has been financially supported through the CONACyT Grants \# 134085 and \# 151494.

\section{REFERENCES}

[1] M. L. Heger, "Further Study of the Sodium Lines in Class B Stars," Lick Observatory Bulletin, Vol. 10, No. 337, 1922, pp. 141-145.

[2] G. H. Herbig, "The Diffuse Interstellar Bands. IV. The Region 4400-6850 A," Astrophysical Journal, Vol. 196, Pt. 1, 1975, pp. 129-160. http://dx.doi.org/10.1086/153400

[3] L. Spitzer, J. F. Drake, E. B. Jenkins, D. C. Morton, J. B. Rogerson and D. G. York, "Spectrophotometric Results from the Copernicus Satellite. IV. Molecular Hydrogen in Interstellar Space,” Astrophysical Journal, Vol. 181, 1973,

\section{p. L116. http://dx.doi.org/10.1086/181197}

[4] E. B. Jenkins, J. F. Drake, D. C. Morton, J. B. Rogerson, L. Spitzer and D. G. York, "Spectrophotometric Results from the Copernicus Satellite. V. Abundances of Molecules in Interstellar Clouds," Astrophysical Journal, Vol. 181, 1973, p. L122. http://dx.doi.org/10.1086/181198

[5] P. Solomon, K. B. Jefferts, A. A. Penzias and R. W. Wilson, "Observation of CO Emission at 2.6 Millimeters from IRC+10216,” Astrophysical Journal, Vol. 163, 1971, p. L53. http://dx.doi.org/10.1086/180665

[6] K. B. Jefferts, A. A. Penzias and R. W. Wilson, "Observation of the CN Radical in the Orion Nebula and W51," Astrophysical Journal, Vol. 161, 1973, p. L87. http://dx.doi.org/10.1086/180576

[7] M. R. Swain, G. Tinetti, G. Vasisht, P. Deroo, et al. "Water, Methane, and Carbon Dioxide Present in the Dayside Spectrum of the Exoplanet HD 209458b," The Astrophysical Journal, Vol. 704, No. 2, 2009, pp. 16161621. http://dx.doi.org/10.1088/0004-637X/704/2/1616

[8] C. R. Masson, "A Stable Acousto-Optical Spectrometer for Millimeter Radio Astronomy," Astronomy and Astrophysics, Vol. 114, 1982, pp. 270-274.

[9] G. W. Schwaab, K. Meyer, H. P. Roeser, P. van der Wal, R. Wattenbach, "A $1 \mathrm{GHz}$ Bandwidth Acousto-Optical Spectrometer for Airborne Submillimetre Astronomy," Journal of Physics E-Scientific Instruments, Vol. 22, No. 7, 1989, pp. 510-514. http://dx.doi.org/10.1088/0022-3735/22/7/018

[10] A. Lecacheux, C. Rosolen, P. Dierich and G. Paubert, "Acousto-Optical Spectrometers for Broadband Millimeter Radioastronomy at I.R.A.M.," International Journal of Infrared and Millimeter Waves, Vol. 14, No. 2, 1993, pp. 169-184. http://dx.doi.org/10.1007/BF02282063

[11] J. Horn, O. Siebertz, F. Schmülling, C. Kunz, R. Schieder, G. Winnewisser, "A $4 \times 1 \mathrm{GHz}$ Array Acousto-Optical Spectrometer," Experimental Astronomy, Vol. 9, No. 1, 1998, pp. 17-38.

[12] T. de Graauw, F. P. Helmich, T. G. Phillips, J. Stutzki, E. Caux, N. D. Whyborn, et al., "The Herschel-Heterodyne Instrument for the Far-Infrared (HIFI)," Astronomy and Astrophysics, Vol. 518, 2010, p. L6. http://dx.doi.org/10.1051/0004-6361/201014698

[13] B. Tercero, J. Cernicharo, J. R. Pardo and J. R. Goicoechea, "A Line Confusion Limited Millimeter Survey of Orion KL. I. Sulfur Carbon Chains," Astronomy and Astrophysics, Vol. 517, 2010, p. A96. http://dx.doi.org/10.1051/0004-6361/200913501

[14] M. Chavez, D. Hughes and LMT Project Team, "The Large Millimeter Telescope and Solar Like Stars, New Quests in Stellar Astrophysics III: A Panchromatic View of Solar-Like Stars, With and Without Planets," M. Chavez, E. Bertone, O. Vega and V. De la Luz, Eds., Proceedings of an International Conference held at Puerto Vallarta, Astronomical Society of the Pacific, San Francisco, 2013, pp. 279-289.

[15] G. Narayanan, M. H. Heyer, C. Brunt, P. F. Goldsmith, R. Snell and D. Li, "The Five College Radio Astronomy Observatory CO Mapping Survey of the Taurus Molecu- 
lar Cloud," The Astrophysical Journal Supplement Series, Vol. 177, No. 1, 2008, pp. 341-361. http://dx.doi.org/10.1086/587786

[16] M. Wyatt, "Evolution of Debris Disks," Annual Review of Astronomy and Astrophysics, Vol. 46, 2008, pp. 339-383. http://dx.doi.org/10.1146/annurev.astro.45.051806.11052 $\underline{5}$

[17] J. Williams and L. Cieza, "Protoplanetary Disks and Their Evolution," Annual Review of Astronomy and Astrophysics, Vol. 49, 2011, pp. 67-117.

http://dx.doi.org/10.1146/annurev-astro-081710-102548

[18] A. Roberge, et al. "High-Resolution Hubble Space Telescope STIS Spectra of CI and CO in the $\beta$ Pictoris Circumstellar Disk," Astrophysical Journal, Vol. 538, No. 2, 2000, pp. 904-910. http://dx.doi.org/10.1086/309157

[19] B. Zuckerman, et al. "Inhibition of Giant-Planet Formation by Rapid Gas Depletion around Young Stars," Nature, Vol. 373, No. 6514, 1995, pp. 494-496. http://dx.doi.org/10.1038/373494a0

[20] W. R. F. Dent, J. Greaves and I. M. Coulson, "CO Emission from Discs around Isolated HAeBe and Vega-Excess Stars," Monthly Notices of the Royal Astronomical Society, Vol. 359, No. 2, 2005, pp. 663-676. http://dx.doi.org/10.1111/j.1365-2966.2005.08938.x

[21] A. Grigorieva, et al. "Survival of Icy Grains in Debris Discs. The Role of Photosputtering," Astronomy \& Astrophysics, Vol. 475, No. 2, 2007, pp. 755-764. http://dx.doi.org/10.1051/0004-6361:20077686

[22] D. Psaltis, "Two-Dimensional Optical Processing Using One-Dimensional Input Devices," Proceedings of the
IEEE, Vol. 72, No. 7, 1984, pp. 962-974. http://dx.doi.org/10.1109/PROC.1984.12952

[23] R. W. Klein and B. D. Cook, "A Unified Approach to Ultrasonic Light Diffraction," IEEE Transactions on Sonics and Ultrasonics, Vol. 14, No. 3, 1967, pp. 123-134. http://dx.doi.org/10.1109/T-SU.1967.29423

[24] T. Bader, "Acousto-Optic Spectrum Analysis. A High Performance Hybrid Technique," Applied Optics, Vol. 18, No. 10, 1979, pp. 1668-1672. http://dx.doi.org/10.1364/AO.18.001668

[25] D. Psaltis and D. Casasent, "Time-and-Space Integrating Spectrum Analyzer," Applied Optics, Vol. 18, No. 19, 1979, pp. 3203-3204. http://dx.doi.org/10.1364/AO.18.003203

[26] P. Kellman, “Time Integrating Optical Processing," Ph.D. Thesis, Stanford University, Stanford, 1979.

[27] T. M. Turpin, "Time Integrating Optical Processing," Proceedings of SPIE, Vol. 154, 1978, pp. 196-203. dx.doi.org/10.1117/12.938255

[28] A. Korpel, “Acousto-Optics,” Marcel Dekker, New York, 1997.

[29] D. R. Pape, O. B. Gusev, S. V. Kulakov and V. V. Molotok, "Chapter 2. Design of Acousto-Optic Deflectors," In: A. P. Goutzoulis and D. R. Pape, Eds., Design and Fabrication of Acousto-Optic Devices, Marcel Dekker, New York, 1994, pp. 69-122.

[30] L. W. J. Kent, "3 GHz Bandwidth Rutile Bragg Cell," Proceedings of SPIE, Vol. 7100, 2008, Article ID: 710027-1. http://dx.doi.org/10.1117/12.803620 